\title{
Lymphopaenia in cardiac arrest patients
}

\author{
Paola Villois ${ }^{1}$, David Grimaldi ${ }^{1}$ Savino Spadaro², Claudia Righy Shinotsuka' ${ }^{1}$ Vito Fontana' ${ }^{1}$ Sabino Scolletta ${ }^{3}$, \\ Federico Franchi ${ }^{3}$, Jean-Louis Vincent ${ }^{1}$, Jacques Creteur ${ }^{1}$ and Fabio Silvio Taccone ${ }^{1 *}$
}

\begin{abstract}
Background: A decrease in circulating lymphocytes has been described as a marker of poor prognosis after septic shock; however, scarce data are available after cardiac arrest (CA). The aim of this study was to evaluate the impact of lymphopaenia after successful cardiopulmonary resuscitation.

Methods: This is a retrospective analysis of an institutional database including all adult CA patients admitted to the intensive care unit (ICU) between January 2007 and December 2014 who survived for at least $24 \mathrm{~h}$. Demographic, CArelated data and ICU mortality were recorded as was lymphocyte count on admission and for the first $48 \mathrm{~h}$. A cerebral performance category score of $3-5$ at 3 months was considered as an unfavourable neurological outcome.

Results: Data from 377 patients were analysed (median age: 62 [IQRs: 52-75] years). Median time to return of spontaneous circulation (ROSC) was 15 [8-25] min and 232 (62\%) had a non-shockable initial rhythm. ICU mortality was $58 \%(n=217)$ and $246(65 \%)$ patients had an unfavourable outcome at 3 months. The median lymphocyte count on

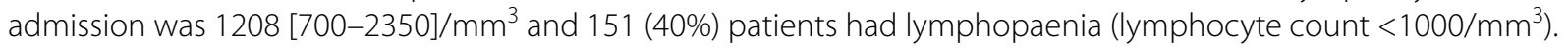
Predictors of lymphopaenia on admission were older age, a shorter time to ROSC, prior use of corticosteroid therapy and high C-reactive protein levels on admission. ICU non-survivors had lower lymphocyte counts on admission than survivors (1100 [613-2317] vs. $\left.1316[891-2395] / \mathrm{mm}^{3} ; p=0.05\right)$ as did patients with unfavourable compared to those with favourable neurological outcomes (1100 [600-2013] vs. 1350 [919-2614]/mm³; $p=0.003$ ). However, lymphopaenia on admission was not an independent predictor of poor outcomes in the entire population, but only among OHCA patients.
\end{abstract}

Conclusions: A low lymphocyte count is common in CA survivors and is associated with poor outcome after OHCA. Keywords: Lymphopaenia, Cardiac arrest, Outcome, Prognosis

\section{Background}

Despite the high mortality rates still associated with sudden cardiac arrest (CA), advances have been made in recent years, in particular to improve the proportion of patients achieving return of spontaneous circulation (ROSC) after cardiopulmonary resuscitation (CPR) [1]. Although the implementation of high-quality CPR and early defibrillation has increased the number of $\mathrm{CA}$ patients being admitted to the hospital, there has been no associated increase in survival rates and neurological recovery among CA survivors $[2,3]$. The high mortality rate observed in this patient population is related

\footnotetext{
*Correspondence: ftaccone@ulb.ac.be

${ }^{1}$ Department of Intensive Care, Erasme Hospital, Université Libre de Bruxelles (ULB), Route de Lennik, 808, 1070 Brussels, Belgium Full list of author information is available at the end of the article
}

to the consequences of the global ischaemia-reperfusion process, the so-called post-cardiac arrest syndrome (PCAS), which is characterised by a systemic inflammatory response that may be involved in the development of myocardial dysfunction, brain injury and multiple organ failure $[3,4]$.

The PCAS is a complex pathophysiological process, which encompasses a generalised activation of immunological and coagulation pathways and has many features common with sepsis [4]. Adrie et al. [4] reported a significant increase in the concentrations of various serum cytokines in CA patients in the first hours after the initial injury, which was more significant in non-survivors than in survivors. Other studies have also shown impaired microcirculatory perfusion and increased plasma endotoxin levels in CA patients, similar to findings in sepsis 
[5, 6]. Interestingly, sepsis is rapidly accompanied by a state of relative immunosuppression, characterised by a reduction in the number of functional immune cells and T-cell dysfunction $[7,8]$, which predicts a poor outcome [9].

Among the different components of the immune response, lymphocytes are essential in the organism's defence against external aggression, by interaction ( $\mathrm{T}$ cells) with antigen-presenting cells and subsequent activation of the immune response ( $\mathrm{B}$ and $\mathrm{T}$ cells), in a complex interplay of cell-to-cell interactions, production of adhesion molecules and modulation of growth factors [10]. Severe lymphopaenia has been described in almost $30 \%$ of patients admitted to the intensive care unit (ICU) with severe sepsis or septic shock and was associated with high plasma levels of tumour necrosis factor- $\alpha$, interleukin (IL)-6 and IL-10 [11]. Lymphopaenia during sepsis was also associated with features of immunosuppression, such as spontaneous hypothermia and an increased risk of hospital-acquired infections, and was an independent predictor of poor outcome $[9,12]$. Persistent lymphopaenia has also been associated with poor outcomes in trauma patients, in particular when an infection occurred during the hospital stay [13]. Although survivors from CA are at high risk of secondary infections, in particular early-onset pneumonia [14], no data are available on the relationship between lymphocyte count and the risk of infection or patient outcome. Thus, the aim of our study was to evaluate the prevalence of lymphopaenia among CA survivors and its association with ICU survival and long-term neurological outcome. We hypothesised that lymphopaenia could be a significant predictor of poor outcome in this setting.

\section{Methods}

\section{Study population}

This study was performed in the Department of Intensive Care at Erasme Hospital. All comatose patients (Glasgow Coma Scale [GCS] score <9) admitted after in-hospital (IHCA) or out-of-hospital (OHCA) CA and surviving for at least $24 \mathrm{~h}$ after the arrest were included in an institutional database (January 2007-December 2014) and considered as eligible for the study. Exclusion criteria were missing data for blood count or lymphocyte count on admission.

\section{Post-resuscitation care}

All comatose CA patients were treated with targeted temperature management (TTM; target body temperature $32-34^{\circ} \mathrm{C}$ ) for $24 \mathrm{~h}$, according to a standardised institutional protocol. Cooling was started immediately after hospital admission using a bolus of cold fluid (in general saline solutions, given as a dose of $20-30 \mathrm{~mL} / \mathrm{kg}$ over
$30 \mathrm{~min}$ ) and a water-circulating blanket device (MediTherm II, Gaymar, USA, or Arctic Sun, Bard, France). Sedation and analgesia consisted of midazolam and morphine, which were adjusted to obtain deep sedation (e.g. severe depression of consciousness during which patients cannot be aroused with repeated or painful stimulation); cisatracurium was administered to control shivering in the induction phase (bolus of $0.15 \mathrm{mg} / \mathrm{kg}$ ) and then given, if needed, by continuous infusion $(1-3 \mathrm{mcg} / \mathrm{kg} /$ min). Rewarming $\left(<0.5^{\circ} \mathrm{C} / \mathrm{h}\right)$ was achieved passively, and sedation/analgesia was discontinued at normothermia $\left(>37^{\circ} \mathrm{C}\right)$.

Patients were kept in a $30^{\circ}$ semi-recumbent position; ventilation was set to target $\mathrm{PaCO}_{2}$ between 35 and $45 \mathrm{mmHg}$ and $\mathrm{SpO}_{2}>94 \%$. Blood glucose was kept between 110 and $150 \mathrm{mg} / \mathrm{dL}$ using a local protocol for continuous insulin infusion; enteral nutrition was allowed. Mean arterial pressure was maintained at least above $65 \mathrm{mmHg}$ using volume resuscitation, noradrenaline and/or dobutamine, when needed. Intra-aortic balloon counterpulsation (IABP) or extracorporeal membrane oxygenation (ECMO) was also used in severe cardiogenic shock.

\section{Data collection}

We collected data on demographics, pre-existing chronic diseases and immunosuppressive treatment, and CPR (initial rhythm, bystander CPR, time to ROSC, total adrenaline dose) in all patients. A complete blood count, including neutrophil and lymphocyte cell counts, and C-reactive protein (CRP) concentration were recorded on ICU admission and during the first $48 \mathrm{~h}$. We also recorded the use of any other immunosuppressive drugs, vasopressors, mechanical ventilation and continuous renal replacement therapy (CRRT) during the ICU stay. Lactate concentrations were also collected on admission. The development of infections during the ICU stay was recorded; survival was recorded at ICU discharge and ICU length of stay was noted. Neurological evaluation at 3 months after CA was assessed using the cerebral performance category score (CPC; $1=$ no or mild neurological disability, 2 = moderate neurological disability, $3=$ severe neurological impairment, $4=$ vegetative state, $5=$ death). The CPC evaluation was assessed during follow-up visits or by telephone interview with the general practitioner.

\section{Definitions}

Lymphopaenia was defined as an absolute lymphocyte count of $<1000 / \mathrm{mm}^{3}$ cells; severe lymphopaenia was defined as an absolute lymphocyte count of $<500 / \mathrm{mm}^{3}$ cells [15]. Patients were subsequently divided into four groups according to the lymphocyte levels during the 
study period: "group 1" included patients with persistent lymphopaenia throughout the $48 \mathrm{~h}$; "group 2" included patients with lymphopaenia on admission but with normal lymphocyte counts at $48 \mathrm{~h}$; "group 3" included those who had normal lymphocyte counts throughout the 48-h study period; and "group 4" included those with normal lymphocyte counts on admission but who had developed lymphopaenia by $48 \mathrm{~h}$.

Favourable 3-month neurological outcome was defined as a CPC of 1-2 at 3 months and unfavourable neurological outcome as a CPC of 3-5. The diagnosis of infection was made according to the CDC/NHSN criteria [16]. Acute kidney injury (AKI) was defined as a daily urine output $<0.5 \mathrm{~mL} / \mathrm{kg} / \mathrm{h}$ and/or an increase in serum creatinine level by at least $0.3 \mathrm{mg} / \mathrm{dL}$ or $>1.5$ times increase from baseline values, as previously reported [17]. Shock was defined as the need for vasopressor agents for more than $6 \mathrm{~h}$.

\section{Statistical analysis}

Statistical analyses were performed using the SPSS 24.0 for Windows NT software package (SPSS Inc, Chicago, IL, USA). Descriptive statistics were computed for all study variables. The Kolmogorov-Smirnov test was used and histograms and normal-quantile plots were examined to verify the normality of distribution of continuous variables. Discrete variables were expressed as counts (percentage) and continuous variables as means $\pm \mathrm{SD}$ or median (25th to 75th percentiles). Demographics and clinical differences between groups were assessed using the Chi-square test, Fisher's exact test, Student's $t$ test or Mann-Whitney $U$ test, as appropriate. The significance of differences among the four groups was analysed using two-way (time and group) analysis of variance for repeated measures (ANOVA), followed by Bonferroni post hoc analysis. Multivariable logistic regression analysis with lymphopaenia on admission as the dependent variable was performed in all patients; co-linearity between variables was excluded prior to modelling; only variables associated with a higher risk of ICU mortality $(p<0.2)$ on a univariate basis were introduced in the multivariable model. Odds ratios (OR) with 95\% confidence intervals $(\mathrm{CI})$ were computed. The same analysis was then performed to identify independent predictors of survival and neurological outcome and for all measured outcome considering only IHCA or OHCA patients. Also, an additional analysis on the independent predictors of lymphopaenia on admission with the exclusion of patients under corticosteroids or other immunosuppressive therapies was performed. A $p$ value $<0.05$ was considered as statistically significant.

\section{Results}

Of the 424 eligible patients admitted during the study period, 23 died within the first $24 \mathrm{~h}$ and 24 were excluded for missing data, so that 377 were included in the final analysis. The main characteristics of the study population (age 62 [52-72] years-OHCA 56\%) are given in Table 1. Median time to ROSC was 15 min and 232 (62\%) patients had a non-shockable initial rhythm; 150 (40\%) patients had a non-cardiac origin of arrest. ICU mortality was $58 \%(n=217)$ and 131 patients $(38 \%)$ had a favourable neurological outcome.

\section{Lymphocyte count}

The median white blood cell count on the day of admission was $12,200[8700-16,775] / \mathrm{mm}^{3}$, including a lymphocyte count of $1100[600-2208] / \mathrm{mm}^{3}$. The lymphocyte count was lower in patients previously treated with immunosuppressive agents $(n=88)$ compared to the other patients $\left(800[445-1989] / \mathrm{mm}^{3}\right.$ vs. 1290 [765$\left.2439] / \mathrm{mm}^{3} ; p=0.004\right)$, in patients with IHCA compared to those with OHCA (946 [583-1450]/ $\mathrm{mm}^{3}$ vs. 1637 $\left.[900-3120] / \mathrm{mm}^{3} ; p<0.001\right)$ and in patients with nonshockable rhythms compared to those with shockable

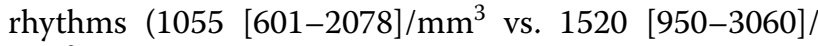
$\mathrm{mm}^{3} ; p<0.001$-Fig. 1$)$.

When grouped according to the change in lymphocyte count during the 48 -h period, 112 patients (30\%) were in group 1, $39(25 \%)$ in group 2, 86 (23\%) in group 3 and 140 (37\%) in group 4. The proportions of patients receiving immunosuppressive therapy, of non-survivors, of patients with unfavourable outcome and of patients developing an infection during the ICU stay were significantly higher in group 1 than in the other groups (Fig. 2).

\section{Lymphopaenia}

A total of 151 (40\%) patients had lymphopaenia, which was severe in 48 patients. Patients with lymphopaenia on admission were older, had a shorter time to ROSC and were more likely to have had IHCA, a non-cardiac aetiology of CA, bystander CPR and a non-shockable initial rhythm than patients without lymphopaenia (Table 1). Comorbid hypertension, diabetes and immunosuppressive therapy were more common in patients with lymphopaenia than in those without. Patients with lymphopaenia on admission also had a lower neutrophil count and haemoglobin concentration on admission and higher CRP concentrations. Lymphopaenic patients more often developed an infection and AKI during the study period and more frequently required vasopressors and CRRT. 
Table 1 Characteristics of study population according to the presence of lymphopaenia on admission

\begin{tabular}{|c|c|c|c|c|}
\hline & All $(n=377)$ & Lymphopaenia $(n=151)$ & No lymphopaenia $(n=226)$ & $p$ value \\
\hline Age (years) & $62[52-75]$ & $69[54-78]$ & $59[51-71]$ & $<0.001$ \\
\hline Weight (kg) & $76[67-85]$ & 75 [67-82] & 77 [67-88] & 0.22 \\
\hline Male, $n(\%)$ & $263(70)$ & $105(70)$ & $158(70)$ & 0.51 \\
\hline Witnessed CA, n (\%) & $318(84)$ & $132(87)$ & $186(82)$ & 0.12 \\
\hline Bystander CPR, $n(\%)$ & $249(66)$ & $110(73)$ & $139(62)$ & 0.02 \\
\hline Time to ROSC (min) & $15[8-25]$ & $10[5-21]$ & 18 [10-27] & $<0.001$ \\
\hline Adrenaline (mg) & $3[2-6]$ & $3[1-5]$ & $4[2-7]$ & $<0.001$ \\
\hline Out-of-hospital CA, $n$ (\%) & $211(56)$ & $60(40)$ & $151(67)$ & $<0.001$ \\
\hline Cardiac origin of arrest, $n(\%)$ & $227(60)$ & $80(53)$ & $147(65)$ & 0.01 \\
\hline Shockable rhythm, n (\%) & $145(38)$ & $41(27)$ & $104(46)$ & $<0.001$ \\
\hline \multicolumn{5}{|l|}{ Comorbidities } \\
\hline Chronic heart failure, $n(\%)$ & $81(21)$ & $37(25)$ & $44(19)$ & 0.15 \\
\hline Hypertension, n (\%) & $171(45)$ & $78(52)$ & $93(41)$ & 0.03 \\
\hline Coronary artery disease, $n(\%)$ & $164(44)$ & $62(41)$ & $102(45)$ & 0.25 \\
\hline Diabetes, $n(\%)$ & $92(24)$ & $48(32)$ & $44(19)$ & 0.01 \\
\hline COPD/asthma, $n(\%)$ & $66(18)$ & $31(21)$ & $35(15)$ & 0.13 \\
\hline Neurological disease, $n$ (\%) & $64(17)$ & $31(21)$ & $32(14)$ & 0.05 \\
\hline Chronic renal disease, $n(\%)$ & $67(18)$ & $36(24)$ & $31(14)$ & 0.008 \\
\hline Liver cirrhosis, $n$ (\%) & $22(6)$ & $14(9)$ & $8(4)$ & 0.02 \\
\hline HIV, $n(\%)$ & $1(0)$ & $1(1)$ & 0 & 0.40 \\
\hline Corticosteroid therapy, $n(\%)$ & $73(19)$ & $41(27)$ & $32(14)$ & 0.001 \\
\hline Other immunosuppressive agents, $n(\%)$ & $15(4)$ & $11(7)$ & $4(2)$ & 0.008 \\
\hline \multicolumn{5}{|l|}{ Initial blood analysis } \\
\hline White blood cells count $\left(/ \mathrm{mm}^{3}\right)$ & $12,200[9000-16,700]$ & $10,700[7700-14,500]$ & $13,100[9925-17,475]$ & 0.06 \\
\hline Neutrophils $\left(/ \mathrm{mm}^{3}\right)$ & $9400[6300-13,500]$ & $9100[6500-13,200]$ & $9915[5947-13,700]$ & $<0.001$ \\
\hline Haemoglobin (g/dL) & $12.0[9.9-13.7]$ & $10.6[8.9-12.9]$ & $12.8[10.8-14.1]$ & 0.04 \\
\hline Platelets $\left(* 10^{3} / \mathrm{mm}^{3}\right)$ & 193 [127-264] & 158 [100-230] & 210 [147-285] & $<0.001$ \\
\hline C-reactive protein (mg/dL) & $9[2-52]$ & 29 [4-93] & $5[2-22]$ & $<0.001$ \\
\hline Lactate (mEq/L) & $4.3[2.8-7.9]$ & $4.1[2.6-7.8]$ & $4.5[2.9-7.9]$ & 0.20 \\
\hline \multicolumn{5}{|l|}{ During ICU stay } \\
\hline Infection, $n(\%)$ & $215(57)$ & $100(66)$ & $115(51)$ & 0.002 \\
\hline IABP, $n(\%)$ & $27(7)$ & $6(4)$ & $21(9)$ & 0.04 \\
\hline $\mathrm{ECMO}, n(\%)$ & $51(14)$ & $23(15)$ & $28(12)$ & 0.26 \\
\hline Shock, $n(\%)$ & $205(54)$ & $90(60)$ & $115(51)$ & 0.06 \\
\hline Acute kidney injury, $n$ (\%) & $228(60)$ & $100(66)$ & $128(57)$ & 0.04 \\
\hline CRRT, $n(\%)$ & $57(15)$ & $30(20)$ & $27(12)$ & 0.03 \\
\hline Vasopressor therapy, $n(\%)$ & $274(73)$ & $120(79)$ & $154(68)$ & 0.01 \\
\hline Dobutamine therapy, n (\%) & $200(53)$ & $85(56)$ & $115(51)$ & 0.18 \\
\hline \multicolumn{5}{|l|}{ Outcomes } \\
\hline Total ICU stay (days) & $4[2-8]$ & $4[2-8]$ & $4[2-8]$ & 0.93 \\
\hline ICU mortality, n (\%) & $217(58)$ & $95(63)$ & $122(54)$ & 0.05 \\
\hline Hospital mortality, n (\%) & $235(62)$ & $103(68)$ & $132(58)$ & 0.03 \\
\hline Favourable outcome at 3 months, $n(\%)$ & $131(35)$ & $43(28)$ & $88(39)$ & 0.02 \\
\hline
\end{tabular}

$C A$ cardiac arrest, $C P R$ cardiopulmonary resuscitation, $R O S C$ return of spontaneous circulation, COPD chronic obstructive pulmonary disease, IABP intra-aortic balloon pump counterpulsation, ECMO extracorporeal membrane oxygenation, CRRT continuous renal replacement therapy, ICU intensive care unit, HIV human immunodeficiency virus

\section{Lymphopaenia, survival and neurological outcome}

ICU non-survivors had a lower lymphocyte count on admission than ICU survivors $\left(1100[613-2317] / \mathrm{mm}^{3}\right.$ vs. 1316 [891-2395]/ $\left.\mathrm{mm}^{3} ; p=0.048\right)$. The non-survivors were older, more frequently had unwitnessed CA, had a longer time to ROSC and had a higher occurrence of 


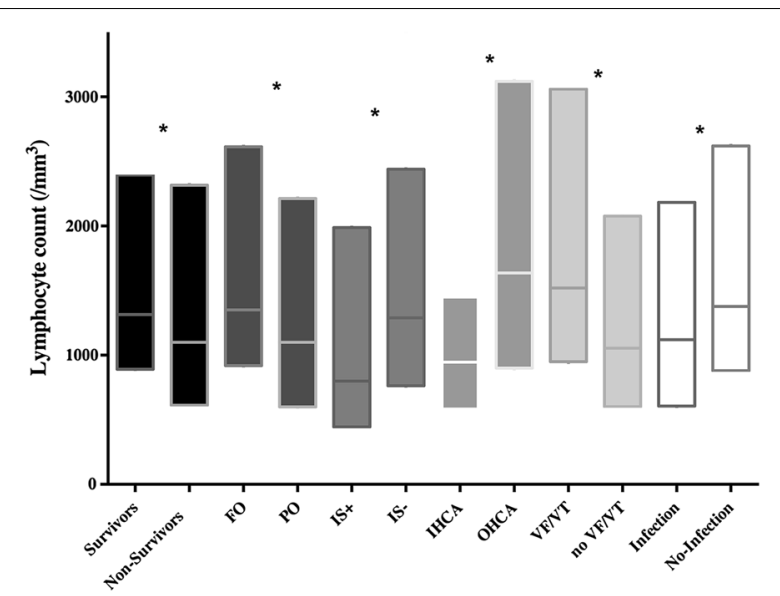

Fig. 1 Differences in lymphocyte counts on admission among survivors versus non-survivors; patients with favourable (FO) versus unfavourable neurological outcome (UO); patients receiving immunosuppressive agents (IS+) versus others (IS-); patients with inhospital (IHCA) versus out-of-hospital cardiac arrest (OHCA); patients with shockable rhythms (VFNT) versus others (no VFNT); patients who developed infection versus those without infection. Data are presented as median and 25th (lower limit) and 75th percentiles (upper limit). * $p<0.05$ for lymphocyte count

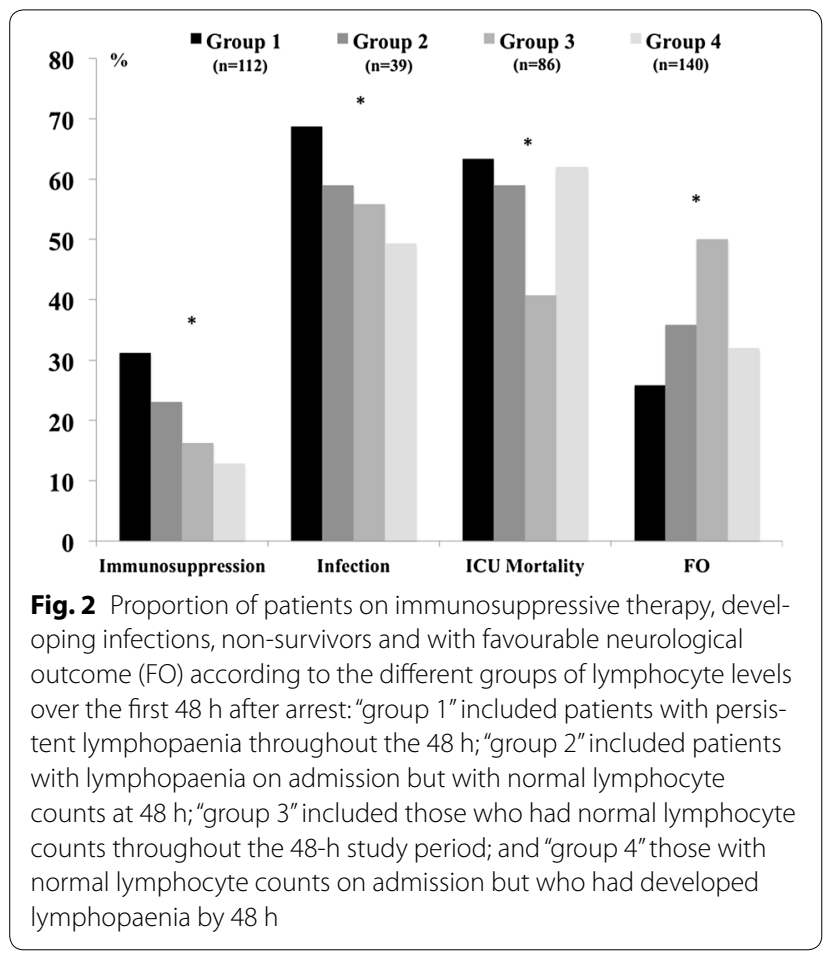

non-cardiac origin of arrest and non-shockable rhythms than survivors (Table 2). Non-survivors had higher lactate levels on admission and more frequently had shock or AKI during the ICU stay than survivors. These patterns were similar in patients with unfavourable compared to those with favourable outcomes (Table 2).

\section{Multivariable analyses to predict lymphopaenia}

In a multivariable logistic regression analysis, older age, a shorter time to ROSC, comorbid use of corticosteroid therapy and high CRP levels on admission were independently associated with the presence of lymphopaenia on admission (Table 3); IHCA was not associated with the occurrence of lymphopenia (OR 1.741 [ -3.032 to 49.710]; $p=0.083$ ). In IHCA patients, older age, a shorter time to ROSC, high white blood cells count and CRP levels on admission and the use of corticosteroids were independently associated with the presence of lymphopaenia on admission (Additional file 1: Supplemental Tables 1 and 5). In OHCA patients, a shorter time to ROSC, high white blood cells and CRP levels as well as low platelet count on admission were independently associated with the presence of lymphopaenia on admission (Additional file 1: Supplemental Tables 1 and 5). Finally, older age, a shorter time to ROSC, the presence of a non-shockable rhythm, high white blood cells count and CRP levels or low platelets count on admission and the use of corticosteroids were independently associated with the presence of lymphopaenia on admission in those patients without corticosteroids or immunosuppressive therapy (Additional file 1: Supplemental Tables 4 and 8).

\section{Multivariable analyses to predict ICU mortality}

Using the same statistical approach, older age, the absence of bystander CPR, a non-shockable initial rhythm, a non-cardiac aetiology of the arrest, high blood lactate levels on admission and the use of vasopressors were independent predictors of ICU mortality (Table 4), but lymphopaenia was not (OR 1.367 [0.787-2.567; $p=0.26)$. In IHCA patients, the absence of bystander CPR, a non-shockable rhythm, high epinephrine dose and the development of shock were independently associated with ICU mortality (Additional file 1: Supplemental Tables 2, 6 and 7), but not lymphopaenia (OR 1.319 [0.708-2.458]; $p=0.38$ ). In OHCA patients, older age, the absence of bystander CPR, a non-cardiac origin of the arrest, a non-shockable rhythm and high blood lactate levels on admission were independent predictors of ICU mortality (Additional file 1: Supplemental Tables 3, 6 and 7); lymphopaenia was not associated with ICU mortality (OR $1.912[0.876-3.621] ; p=0.09)$.

\section{Multivariable analyses to predict poor long-term neurological outcome}

In the multivariable analysis, older age, the absence of bystander CPR and a non-shockable initial rhythm were independent predictors of an unfavourable neurological 
Table 2 Characteristics of study population according to ICU survival and long-term neurological outcome

\begin{tabular}{|c|c|c|c|c|}
\hline & ICU survivors $(n=160)$ & ICU non-survivors $(n=217)$ & $\begin{array}{l}\text { Favourable outcome } \\
(n=131)\end{array}$ & $\begin{array}{l}\text { Unfavourable outcome } \\
(n=246)\end{array}$ \\
\hline Age (years) & $59[49-71]$ & $66[54-78]^{*}$ & $58[50-70]$ & $66[53-77]^{*}$ \\
\hline Weight (kg) & 77 [70-85] & 75 [65-85] & $78[70-85]$ & 75 [65-85] \\
\hline Male, $n(\%)$ & $118(74)$ & $145(67)$ & $97(74)$ & $166(67)$ \\
\hline Witnessed CA, $n(\%)$ & $144(90)$ & $174(80)^{*}$ & $118(90)$ & $200(80)^{*}$ \\
\hline Bystander CPR, $n(\%)$ & $122(76)$ & $127(59)^{*}$ & $101(77)$ & $148(60)^{*}$ \\
\hline Time to ROSC (min) & $12[5-20]$ & $18[10-25]^{*}$ & $12[5-20]$ & $17[10-25]^{*}$ \\
\hline Adrenaline (mg) & $2[1-4]$ & $4[2-7]^{*}$ & $2[1-4]$ & $4[2-6]^{*}$ \\
\hline Out-of-hospital CA, n (\%) & $86(54)$ & $125(57)$ & $74(56)$ & $137(56)$ \\
\hline Cardiac origin of arrest, $n(\%)$ & $110(69)$ & $117(54)^{*}$ & $102(78)$ & $133(54)^{*}$ \\
\hline Shockable rhythm, n (\%) & $89(56)$ & $56(26)^{*}$ & $82(63)$ & $63(26)^{*}$ \\
\hline \multicolumn{5}{|l|}{ Comorbidities } \\
\hline Chronic heart failure, $n(\%)$ & $33(21)$ & $48(22)$ & $27(21)$ & $54(22)$ \\
\hline Hypertension, $n$ (\%) & $76(48)$ & $95(44)$ & $60(46)$ & $111(45)$ \\
\hline $\begin{array}{l}\text { Coronary artery disease, } \\
n(\%)\end{array}$ & $67(42)$ & $97(45)$ & $54(41)$ & $110(45)$ \\
\hline Diabetes, $n(\%)$ & $35(22)$ & $57(26)$ & $25(19)$ & $67(27)$ \\
\hline COPD/asthma, n (\%) & $26(16)$ & $40(18)$ & $19(15)$ & $47(19)$ \\
\hline Neurological disease, $n(\%)$ & $22(14)$ & $42(19)$ & $13(10)$ & $51(21)^{*}$ \\
\hline Chronic renal disease, $n(\%)$ & $26(16)$ & $41(19)$ & $20(15)$ & $47(19)$ \\
\hline Liver cirrhosis, $n(\%)$ & $7(4)$ & $15(7)$ & $4(3)$ & $18(7)$ \\
\hline$H I V, n(\%)$ & $1(1)$ & - & $1(1)$ & - \\
\hline Corticosteroid therapy, n (\%) & $25(16)$ & $48(22)$ & $20(15)$ & $53(22)$ \\
\hline $\begin{array}{l}\text { Immunosuppressive agents, } \\
n(\%)\end{array}$ & $5(3)$ & $10(5)$ & $3(2)$ & $12(5)$ \\
\hline $\begin{array}{l}\text { Lactate on admission } \\
(\mathrm{mEq} / \mathrm{L})\end{array}$ & $3.8[2.4-5.7]$ & $4.9[3.2-8.7]^{*}$ & $3.9[2.5-5.7]$ & $4.6[3-8.5]^{*}$ \\
\hline \multicolumn{5}{|l|}{ During ICU stay } \\
\hline Infection, $n(\%)$ & $105(66)$ & $110(51)^{*}$ & $83(63)$ & $132(54)$ \\
\hline IABP, $n(\%)$ & $9(6)$ & $18(8)$ & $7(5)$ & $20(8)$ \\
\hline $\mathrm{ECMO}, n(\%)$ & $19(12)$ & $32(15)$ & $18(14)$ & $33(13)$ \\
\hline Shock, $n(\%)$ & $68(43)$ & $137(63)^{*}$ & $58(44)$ & $147(60)^{*}$ \\
\hline Acute kidney injury, n (\%) & $81(51)$ & $147(68)^{*}$ & $65(50)$ & $163(66)^{*}$ \\
\hline CRRT, $n(\%)$ & $23(14)$ & $34(16)$ & $19(15)$ & $38(15)$ \\
\hline Vasopressor therapy, $n$ (\%) & $99(62)$ & $175(81)^{*}$ & $81(62)$ & $193(78)^{*}$ \\
\hline Dobutamine therapy, $n(\%)$ & $81(51)$ & $119(55)$ & $66(50)$ & $134(54)$ \\
\hline Total ICU stay & $6[3-11]$ & $2[2-5]^{*}$ & $5[3-11]$ & $3[2-5]^{*}$ \\
\hline
\end{tabular}

$C A$ cardiac arrest, $C P R$ cardiopulmonary resuscitation, $R O S C$ return of spontaneous circulation, COPD chronic obstructive pulmonary disease, IABP intra-aortic balloon pump counterpulsation, ECMO extracorporeal membrane oxygenation, CRRT continuous renal replacement therapy, ICU intensive care unit, HIV human immunodeficiency virus

${ }^{*} p<0.05$ in survivors versus non-survivors $\mathrm{OR}$ favourable versus unfavourable outcome

outcome (Table 4); lymphopaenia was not significantly associated with unfavourable outcome either (OR 1.228 [0.723-3.167; $p=0.28$ ). In IHCA patients, the absence of bystander CPR and a non-shockable rhythm were independently associated with UO (Additional file 1: Supplemental Tables 2, 6 and 7), but not lymphopaenia (OR 1.219 [0.719-2.973]; $p=0.36$ ). In OHCA patients, older age, a non-cardiac origin of the arrest, lymphopaenia on admission and high epinephrine doses were independent predictors of UO (Additional file 1: Supplemental Tables 3, 6 and 7).

\section{Discussion}

In this study, $40 \%$ of post-CA patients had lymphopaenia, and in one-third of them, this persisted through the first $48 \mathrm{~h}$ after admission. Predictors of lymphopaenia on 
Table 3 Multivariable regression analysis to identify independent predictors of lymphopaenia on admission

\begin{tabular}{lllll}
\hline & \multicolumn{3}{l}{ Lymphopaenia on admission } \\
\cline { 3 - 6 } & $\boldsymbol{p}$ value & OR & \multicolumn{2}{c}{$\mathbf{9 5 \%}$ Cl for OR } \\
\cline { 4 - 6 } & & & Lower & Upper \\
\hline Age (years) & 0.012 & 1.023 & 1.005 & 1.042 \\
Time to ROSC (min) & 0.001 & 0.960 & 0.936 & 0.984 \\
Previous corticosteroid therapy & 0.048 & 2.040 & 1.007 & 4.131 \\
CRP (mg/dL) & 0.007 & 1.005 & 1.002 & 1.009 \\
\hline
\end{tabular}

Hosmer and Lemeshow goodness-of-fit test Chi-squared $=4.93(p=0.76)$. This model has a $69 \%$ correct classification ( $48 \%$ for lymphopenia and $83 \%$ for nonlymphopenia)

$R O S C$ return of spontaneous circulation, $C R P C$-reactive protein

admission were older age, a shorter resuscitation time, a history of corticosteroid therapy and high levels of the inflammatory marker (e.g. CRP). Lymphopaenia was more frequent in patients with poor outcome but was not an independent predictor of mortality or unfavourable neurological outcome, unless in the subgroup of OHCA patients.

The occurrence of lymphopaenia and its impact on survivors of CA has not been well studied. In a pig model of prolonged $\mathrm{CA}$ and $\mathrm{CPR}, \mathrm{Gu}$ et al. reported a high degree of splenic lymphocyte apoptosis, which was initiated by activation of the $\mathrm{Bcl}-2 / \mathrm{Bax}$ mitochondrial pathway [18].
These authors also described a reduction in $\mathrm{CD} 4^{+} / \mathrm{CD} 8^{+}$ $\mathrm{T}$ lymphocytes and a shift in these cells from an antiinflammatory (Th-2) to a pro-inflammatory (T-helper [Th]-1) state in the myocardium $[19,20]$. In mice submitted to global cerebral ischaemia secondary to CA within $3 \mathrm{~h}$ after resuscitation, Deng et al. [21] showed the presence of infiltrating peripheral $\mathrm{CD} 4^{+} \mathrm{T}$ lymphocytes in the brain. These experimental data indicate that early disturbances of immunological function after ROSC are associated with an increased production of inflammatory mediators and lymphocyte apoptosis, as well as an intense cardiac and neuroimmune response in which infiltrating $\mathrm{T}$ cells may play a key role. Few data are available in humans. In 50 OHCA patients, Venet et al. [22] reported a moderate decrease in the number of circulating $\mathrm{CD} 4^{+} \mathrm{T}$ lymphocytes although total lymphocyte count was normal. Nevertheless, the high mortality rate in that study $(90 \%)$ prevented a comparison of lymphocyte levels in survivors and non-survivors. Indeed, as clinical studies have demonstrated that PCAS is characterised by high plasma cytokine and endotoxin levels, future studies should better characterise how these abnormalities may alter lymphocyte sub-populations and function in CA survivors and whether these changes are reversible or can be influenced by therapeutic interventions [23, 24].

Lymphopaenia has been widely described in patients with sepsis [12, 25, 26]. After an initial predominant

Table 4 Multivariable regression analysis to identify independent predictors of ICU mortality and unfavourable neurological outcome at 3 months after cardiac arrest

\begin{tabular}{|c|c|c|c|c|}
\hline & \multicolumn{4}{|c|}{ ICU mortality } \\
\hline & \multirow[t]{2}{*}{$p$ value } & \multirow[t]{2}{*}{ OR } & \multicolumn{2}{|c|}{$95 \% \mathrm{Cl}$ for OR } \\
\hline & & & Lower & Upper \\
\hline Age (years) & 0.001 & 1.042 & 1.033 & 1.055 \\
\hline Bystander CPR & 0.006 & 0.441 & 0.218 & 0.755 \\
\hline Non-cardiac aetiology & 0.015 & 2.069 & 1.345 & 4.568 \\
\hline Non-shockable rhythm & 0.001 & 2.687 & 1.876 & 4.325 \\
\hline Vasopressor use & 0.02 & 2.142 & 1.356 & 8.567 \\
\hline \multirow[t]{4}{*}{ Lactate on admission (mEq/L) } & 0.003 & 1.113 & 1.096 & 1.301 \\
\hline & \multicolumn{4}{|c|}{ Unfavourable neurological outcome } \\
\hline & \multirow[t]{2}{*}{$p$ value } & \multirow[t]{2}{*}{ OR } & \multicolumn{2}{|c|}{$95 \% \mathrm{Cl}$ for OR } \\
\hline & & & Lower & Upper \\
\hline Age (years) & 0.001 & 1.032 & 1.024 & 1.057 \\
\hline Bystander CPR & 0.008 & 0.337 & 0.219 & 0.785 \\
\hline Non-shockable rhythm & $<0.001$ & 2.177 & 1.765 & 3.987 \\
\hline
\end{tabular}

ICU mortality: Hosmer and Lemeshow goodness-of-fit test Chi-squared $=5.16(p=0.23)$. This model has a $71 \%$ correct classification $(57 \%$ for survivors and $81 \%$ for non-survivors)

Neurological outcome: Hosmer and Lemeshow goodness-of-fit test Chi-squared $=9.18(p=0.33)$. This model has a 73\% correct classification (48\% for good neurological outcome and $82 \%$ for poor neurological outcome) 
pro-inflammatory phase, many septic patients develop persistent immunosuppression, characterised by increased inhibitory receptors on $\mathrm{T}$ cells and antigen-presenting cells, decreased production of pro-inflammatory cytokines, expansion of myeloid-derived suppressor cells, and apoptosis-related loss of $\mathrm{T}$ and B-lymphocytes and dendritic cells $[7,27]$. Induction of lymphocyte apoptosis increased mortality and prevented bacteraemic control in septic animals [28]. The detrimental effects of apoptosis are not only related to the severe loss of immune cells but also the impact that apoptotic cell uptake has on the surviving immune cells. As such, uptake of apoptotic cells by monocytes, macrophages and dendritic cells results in immune tolerance and cellular anergy, which is associated with increased IL-10 production and the induction of a Th-2 cell immune phenotype; the net result of these changes is that the surviving phagocytic cells cannot provide adequate defence against infection [29].

In our study, patients with lymphopaenia were older than those without. Lymphopaenia is a common finding in elderly hospitalised patients and has been associated with poor outcome in these patients [30]. There is a significant interaction between the immune system and the ageing process, which may also influence the occurrence of chronic diseases; in particular, thymic demise represents an important phenomenon that can cause a reduction in T-cell count and peripheral proliferation of pre-existing T-cell clones, which can trigger limited immune reactivity damage-associated molecular patterns [31]. As expected, previous therapy with corticosteroids was also an independent determinant of lymphopaenia [32]. Data on the prevalence of lymphopaenia among corticosteroid users are scarce and biased by the presence of concomitant malignancies, administration of chemotherapy or other immunosuppressive drugs. The mechanisms include increased Th-2 cells with reduction in pro-inflammatory circulating cytokines or externalisation of phosphatidylserine, which may trigger cellular apoptosis, in particular of $\mathrm{CD}_{4}^{+}$cells [33]. Nevertheless, we found an association between increasing CRP levels and the risk of lymphopaenia. This is probably due to increased production of IL-6, which has been shown to be an independent predictor of poor outcome in CA patients [34]. In a recent study, the injection of endotoxin in healthy volunteers was associated with systemic inflammation, which triggered the occurrence of lymphopaenia, by an increase in antiinflammatory regulatory $\mathrm{T}$ cells and a relative functional impairment of T-cell cytokine production, despite detectable levels of plasma pro-inflammatory cytokines [35]. Predictors of lymphopaenia in patients without previous immunosuppressive therapy were similar to those of the entire cohort, underlying again their relevance independently of patients' immune status.
Interestingly, a shorter time to ROSC was another variable associated with lymphopaenia. We may have expected that the severity of the ischaemic injury, which is related to the longer duration of CPR, would have been associated with more severe apoptosis of $\mathrm{T}$ cells and have induced a low lymphocyte count in this setting. In stroke, although ischaemic cerebral damage may lead to suppression of peripheral immune responses, which predisposes to infection, no association was found between the extension of the infarct area and functional immune alterations [36]. The higher prevalence of IHCA in the lymphopaenia group with a shorter intervention time and CPR may also explain this finding. Additional studies should evaluate the complex interplay between pre-existing comorbid conditions and acute cerebral injury and the development of altered immune responses in CA patients.

There was no independent association between lymphopaenia and poor outcome. In patients with sepsis and septic shock, a low lymphocyte count was a strong predictor of poor outcome, even better than increased neutrophil cells [12]. Although sepsis has many common features with the PCAS, lymphocyte count and function may play a marginal prognostic role in CA survivors. One possible explanation could be that the severity of the initial anoxic brain injury (i.e. bystander CPR and time to ROSC) was not correlated with the occurrence of lymphopaenia. Moreover, many patients died within the first 2-3 days after arrest because of significant cardiovascular impairment or extended post-anoxic brain injury [37], limiting the impact of lymphopaenia on the occurrence of secondary infections or delayed organ dysfunction. Nevertheless, we observed that in the subgroup of OHCA patients, lymphopaenia on admission was associated with UO. This suggests that factors as the underlying different causes of the arrest between IHCA or OHCA or the management of CPR according to arrest location (i.e. different response time and quality of CPR) are an important determinant of biological biomarkers of poor prognosis and that routine monitor of the lymphocyte count in OHCA patients could be considered. However, future research should focus on a better characterisation of lymphocytes sub-populations, in relationship with other biomarker of "immunosuppression" (i.e. HLA-DR on monocytes) to better understand the potential prognostic and therapeutic role of lymphopaenia in this patients' population.

Our study has some limitations. Firstly, it was a retrospective, single-centre study, which may limit the generalisability of our conclusions; this can, however, also be an advantage, as patients were treated according to a local protocol of PCAS management, thus reducing heterogeneity. Secondly, we did not record the exact time of no flow or the quality of CPR, which may influence the inflammatory response after reperfusion. Thirdly, all 
patients were treated with TTM so that we cannot draw any conclusions on lymphopaenia in normothermic CA patients or on the effects of TTM use, which may blunt the inflammatory response after rewarming, on the impact of lymphopaenia. Forth, causes of CA and lymphopaenia may differ between IHCA and OHCA. Also, it is possible that lymphopaenia may have preceded the arrest in some CA victims, such as in case of IHCA. Although location of arrest was not a significant predictor of lymphopaenia in our study, this might also be due to a lack of power and this question should be further addressed in future larger cohorts. Fifth, some patients, especially if suffering from IHCA, may have sepsis prior to ICU admission, and this could have been a significant trigger to lymphopaenia. Unfortunately, sepsis is widely under-recognised outside the ICU, so that this variable could not be reliably assessed in our database. Finally, we perform a multivariate model to identify variables associated with the occurrence of lymphopaenia, but we did not specifically investigate the lymphocyte count as a continuous variable. Although this might appear more appropriate, clinicians would be more interested in the presence of a "lymphopaenia", its related risk factors and potential consequences rather than considering the absolute lymphocytes count.

\section{Conclusions}

Lymphopaenia is a common finding in CA survivors, in particular among those with an initial non-shockable rhythm, in-hospital CA and previous immunosuppressive therapy. A lower lymphocyte count on admission was associated with poor outcome, but was not an independent predictor of mortality or neurological recovery. Future studies should better characterise the immune response in patients resuscitated after CA to improve understanding of the pathophysiology of these findings and their potential therapeutic interventions.

\section{Additional file}

Additional file 1. This documents includes comparisons between patients with in-hospital (IHCA) and out-of-hospital cardiac arrest (OHCA - Suppl Table 1); analysis of mortality and neurological outcome in IHCA (Suppl Table 2) and OHCA (Suppl Table 3) patients; the characteristics of lymphopenic patients after having excluded those receiving immunosuppressive therapies (Suppl Table 4); a multivariable regression analysis to identify independent predictors of lymphopenia on admission in in-hospital (IHCA) or out-of-hospital (OHCA) cardiac arrest (Suppl Table 5): a multivariable regression analysis to identify independent predictors of ICU outcome in in-hospital (IHCA) or out-of-hospital (OHCA) cardiac arrest (Suppl Table 6); a multivariable regression analysis to identify independent predictors of long-term neurological outcome in in-hospital (IHCA) or out-of-hospital (OHCA) cardiac arrest (Suppl Table 7); a multivariable regression analysis to identify independent predictors of lymphopenia on admission in patients without therapy with corticosteroids or other immunosoppressive drugs (Suppl Table 8).

\section{Abbreviations}

CA: cardiac arrest; CPR: cardiopulmonary resuscitation; CPC: cerebral performance category score; CRRT: continuous renal replacement therapy; ECMO: extracorporeal membrane oxygenation; IABP: intra-aortic balloon counterpulsation; ICU: intensive care unit; IHCA: in-hospital cardiac arrest; OHCA: out-ofhospital cardiac arrest; PCAS: post-cardiac arrest syndrome; ROSC: return of spontaneous circulation; TTM: targeted temperature management.

\section{Authors' contributions}

PV, SSC and FST conceived the study protocol; PV, DG, VF, CRS and FST participated in the design and coordination of the study; PV, VF, CRS and FF were responsible for data collection; DG, JC, JLV and JLV supervised data collection; SSp, SSc, FF, DG and VF participated in data interpretation; FF, PV, DG and SSc carried out the literature search; PV, DG, FST and JLV drafted the present manuscript; FST, DG, JC, JLV and SSC and AK revised the manuscript. All authors read and approved the final manuscript.

\section{Authors' information}

PV, DG, CRS, VF, JLV, JC and FST are from the Department of Intensive Care, Erasme Hospital in Brussels (Belgium). SS is from the Department of Morphological Surgery and Experimental Medicine, Arcispedale Sant'Anna, University of Ferrara (Italy). SS and FF are from the Department of Anesthesia and Intensive Care, Policlinico Santa Maria alle Scotte, University of Siena (Italy).

\section{Author details}

${ }_{1}^{1}$ Department of Intensive Care, Erasme Hospital, Université Libre de Bruxelles (ULB), Route de Lennik, 808, 1070 Brussels, Belgium. ${ }^{2}$ Department of Morphological Surgery and Experimental Medicine, Arcispedale Sant'Anna, Università di Ferrara, Via AldoMoro, 8, 44121 Ferrara, Italy. ${ }^{3}$ Department of Anesthesia and Intensive Care, Policlinico Santa Maria alle Scotte, Universitá di Siena, Viale Bracci, 14, 53100 Siena, Italy.

\section{Acknowledgements}

We thank all our clinical and nurse staff for the great support in our daily practice.

\section{Competing interests}

The authors declare that they have no competing interests.

\section{Availability of data and materials}

Data supporting the study are available and can be requested from the corresponding author.

\section{Consent for publication}

All authors agreed with the final version of the manuscript.

\section{Ethics approval and consent to participate}

The local ethical committee (Comité d'Ethique Hospitalo-Facultaire Erasme-ULB) approved the study, but waived the need for informed consent because of its retrospective nature.

\section{Source of funding}

This study was supported by no funding.

\section{Publisher's Note}

Springer Nature remains neutral with regard to jurisdictional claims in published maps and institutional affiliations.

Received: 3 January 2017 Accepted: 6 August 2017

Published online: 14 August 2017

\section{References}

1. Nadkarni VM, Larkin GL, Peberdy MA, et al. National Registry of Cardiopulmonary Resuscitation Investigators. First documented rhythm and clinical outcome from in-hospital cardiac arrest among children and adults. JAMA. 2006;295:50-7. 
2. Stiell IG, Wells GA, Field B, et al. Advanced cardiac life support in out-ofhospital cardiac arrest. N Engl J Med. 2004;351(7):647-56.

3. Neumar RW, Nolan JP, Adrie C, et al. Post-cardiac arrest syndrome: epidemiology, pathophysiology, treatment, and prognostication: a consensus statement from the International Liaison Committee on Resuscitation (American Heart Association, Australian and New Zealand Council on Resuscitation, European Resuscitation Council, Heart and Stroke Foundation of Canada, Inter American Heart Foundation, Resuscitation Council of Asia, and the Resuscitation Council of Southern Africa); the American Heart Association Emergency Cardiovascular Care Committee; the Council on Cardiovascular Surgery and Anesthesia; the Council on Cardiopulmonary, Perioperative, and Critical Care; the Council on Clinical Cardiology; and the Stroke Council. Circulation. 2008;118:2452-83.

4. Adrie C, Adib-Conquy M, Laurent I, et al. Successful cardiopulmonary resuscitation after cardiac arrest as a "sepsis-like" syndrome. Circulation. 2002;106(5):562-8.

5. Donadello K, Favory R, Salgado-Ribeiro D, et al. Sublingual and muscular microcirculatory alterations after cardiac arrest: a pilot study. Resuscitation. 2011;82(6):690-5.

6. Grimaldi D, Sauneuf B, Guivarch E, et al. High level of endotoxemia following out-of-hospital cardiac arrest is associated with severity and duration of postcardiac arrest shock. Crit Care Med. 2015;43(12):2597-604.

7. Hotchkiss RS, Swanson PE, Freeman BD, et al. Apoptotic cell death in patients with sepsis, shock, and multiple organ dysfunction. Crit Care Med. 1999;27(7):1230-51.

8. Grimaldi $D$, Louis $S$, Pène $F$, et al. Profound and persistent decrease of circulating dendritic cells is associated with ICU-acquired infection in patients with septic shock. Intensive Care Med. 2011;37(9):1438-46.

9. Drewry AM, Samra N, Skrupky LP, Fuller BM, Compton SM, Hotchkiss RS. Persistent lymphopenia after diagnosis of sepsis predicts mortality. Shock. 2014;42(5):383-91.

10. Bermejo-Martin JF, Andaluz-Ojeda D, Almansa R, et al. Defining immunological dysfunction in sepsis: a requisite tool for precision medicine. J Infect. 2016;72(5):525-36.

11. Chung KP, Chang HT, Lo SC, et al. Severe lymphopenia is associated with elevated plasma interleukin-15 levels and increased mortality during severe sepsis. Shock. 2015;43(6):569-75.

12. Drewry AM, Fuller BM, Skrupky LP, Hotchkiss RS. The presence of hypothermia within 24 hours of sepsis diagnosis predicts persistent lymphopenia. Crit Care Med. 2015;43(6):1165-9.

13. Menges T, Engel J, Welters I, et al. Changes in blood lymphocyte populations after multiple trauma: association with posttraumatic complications. Crit Care Med. 1999;27:733-40.

14. Kakavas S, Mongardon N, Cariou A, Gulati A, Xanthos T. Early-onset pneumonia after out-of-hospital cardiac arrest. J Infect. 2015;70(6):553-62.

15. Le Tulzo Y, Pangault C, Gacouin A, et al. Early circulating lymphocyte apoptosis in human septic shock is associated with poor outcome. Shock. 2002;18(6):487-94.

16. Horan TC, Andrus M, Dudeck MA. CDC/NHSN surveillance definition of health care-associated infection and criteria for specific types of infections in the acute care setting. Am J Infect Control. 2008;36:309-32.

17. Mehta RL, Kellum JA, Shah SV, et al. Acute Kidney Injury Network: report of an initiative to improve outcomes in acute kidney injury. Crit Care. 2007;11:R31.

18. Gu W, Zhang Q, Yin W, Li C. Caspase-3-mediated splenic lymphocyte apoptosis in a porcine model of cardiac arrest. Am J Emerg Med. 2014;32(9):1027-32.

19. GuW, Li CS, Yin WP, et al. Expression imbalance of transcription factors GATA-3 and T-bet in post-resuscitation myocardial immune dysfunction in a porcine model of cardiac arrest. Resuscitation. 2013;84(6):848-53.
20. Gu W, Li CS, Yin WP, Hou X, Zhang D. Effects of Shen-Fu injection on the expression of T-cell specific transcription factors T-bet/GATA-3 in porcine postresuscitation lung injury. Evid Based Complement Altern Med. 2013;2013:464650.

21. Deng G, Carter J, Traystman RJ, Wagner DH, Herson PS. Pro-inflammatory T-lymphocytes rapidly infiltrate into the brain and contribute to neuronal injury following cardiac arrest and cardiopulmonary resuscitation. J Neuroimmunol. 2014;274(1-2):132-40.

22. Venet F, Cour M, Demaret J, Monneret G, Argaud L. Decreased monocyte HLA-DR expression in patients after non-shockable out-of-hospital cardiac arrest. Shock. 2016;46(1):33-6.

23. Timmermans K, Kox M, Gerretsen J, et al. The involvement of dangerassociated molecular patterns in the development of immunoparalysis in cardiac arrest patients. Crit Care Med. 2015;43(11):2332-8.

24. Beurskens CJ, Horn J, de Boer AM, et al. Cardiac arrest patients have an impaired immune response, which is not influenced by induced hypothermia. Crit Care. 2014;18(4):R162.

25. Heffernan DS, Monaghan SF, Thakkar RK, Machan JT, Cioffi WG, Ayala A. Failure to normalize lymphopenia following trauma is associated with increased mortality, independent of the leukocytosis pattern. Crit Care. 2012;16(1):R12.

26. de Jager CP, van Wijk PT, Mathoera RB, de Jongh-Leuvenink J, van der Poll T, Wever PC. Lymphocytopenia and neutrophil-lymphocyte count ratio predict bacteremia better than conventional infection markers in an emergency care unit. Crit Care. 2010;14(5):R192.

27. Boomer JS, To K, Chang KC, et al. Immunosuppression in patients who die of sepsis and multiple organ failure. JAMA. 2011;306(23):2594-605.

28. Hotchkiss RS, Chang KC, Swanson PE, et al. Caspase inhibitors improve survival in sepsis: a critical role of the lymphocyte. Nat Immunol. 2000;1(6):496-501.

29. Hotchkiss RS, Monneret G, Payen D. Sepsis-induced immunosuppression: from cellular dysfunctions to immunotherapy. Nat Rev Immunol. 2013;13(12):862-74.

30. Rubio-Rivas M, Formiga F, Grillo S, Gili F, Cabrera C, Corbella X. Lymphopenia as prognostic factor for mortality and hospital length of stay for elderly hospitalized patients. Aging Clin Exp Res. 2016;28:271-7.

31. Youm YH, Kanneganti TD, Vandanmagsar B, et al. The Nlrp3 inflammasome promotes age-related thymic demise and immunosenescence. Cell Rep. 2012;1(1):56-68.

32. Yao Z, DuBois DC, Almon RR, Jusko WJ. Pharmacokinetic/pharmacodynamic modeling of corticosterone suppression and lymphocytopenia by methylprednisolone in rats. J Pharm Sci. 2008;97(7):2820-32.

33. Jetzek-Zader M, Gudowius S, Feyen O, Stevens M, Lipfert P, Niehues T. A single intravenous dose of prednisolone induces phosphatidylserine externalization, loss of surface marker expression and a 24-h net increase in human peripheral blood lymphocytes ex vivo. Rheumatol Int. 2007;27(7):667-73.

34. Vaahersalo J, Skrifvars MB, Pulkki K, et al. Admission interleukin-6 is associated with post resuscitation organ dysfunction and predicts longterm neurological outcome after out-of-hospital ventricular fibrillation. Resuscitation. 2014;85(11):1573-9.

35. Ronit A, Plovsing RR, Gaardbo JC, et al. Inflammation-induced changes in circulating t-cell subsets and cytokine production during human endotoxemia. J Intensive Care Med. 2017;32(1):77-85.

36. Klehmet J, Harms H, Richter M, et al. Stroke-induced immunodepression and post-stroke infections: lessons from the preventive antibacterial therapy in stroke trial. Neuroscience. 2009;158(3):1184-93.

37. Lemiale V, Dumas F, Mongardon N, et al. Intensive care unit mortality after cardiac arrest: the relative contribution of shock and brain injury in a large cohort. Intensive Care Med. 2013;39:1972-80. 